\title{
State of the art of Trust and Reputation Systems in E- Commerce Context
}

\author{
Hasnae RAHIMI, Hanan EL BAKKALI \\ Information Security Research Team, \\ University Mohamed V Rabat ENSIAS, Rabat, Morocco.
}

\begin{abstract}
This article proposes in depth comparative study of the most popular, used and analyzed Trust and Reputation System (TRS) according to the trust and reputation literature and in terms of specific trustworthiness criteria. This survey is realized relying on a selection of trustworthiness criteria that analyze and evaluate the maturity and effectiveness of TRS. These criteria describe the utility, the usability, the performance and the effectiveness of the TRS. We also provide a summary table of the compared TRS within a detailed and granular selection of trust and reputation aspects.
\end{abstract}

Keywords: Reputation, Trust, Trust and Reputation System, ECommerce, Survey, Trustworthiness.

\section{Introduction}

Open electronic markets, online collaboration systems, distributed peer-to-peer applications, online social media require the establishment of mutual trust between service providers and service consumers. In fact, the major concerns of web-based services especially e-commerce applications is to overcome the inherent uncertainties and untrustworthiness risks and enhance the system's robustness and resistance against fraudulent users and distrustful ones. Besides, e-commerce platforms aim at adopting the most efficient approach that helps detect and analyze users' intentions in order to reveal and understand deceitful ones. Otherwise, the underlying purpose of ecommerce services which is to maximize the profit and the rate of purchase, would be threatened and deteriorated by fraudulent and ill-intentioned users.

For this reason, Recommender Systems such as Trust and Reputation Systems (TRS), provide essential input for computational trust so as to predict future behaviors of peers basing on the past actions of a peer [1]. In a reputation network, information about these actions can also be received from other members of a reputation network who have transacted with the peer. However, the credibility of this third-party information must be critically assessed. The underlying goal in all reputation systems is to predict a peer's future transactions taking into account his past actions and applying algorithms relying on probabilities approaches.

To gather these first-hand transactions is a tedious and costly task especially when involving the malicious and fraudulent peers. To overcome this limitation, users share their experiences through the reputation system, which aims to detect and effectively isolate misbehaving customers and users. Indeed, e-commerce users refer to this shared information as recommendations on which they rely in order to make the right purchase decision. As no user in e-commerce environment is fully trusted, recommendations and reviews credibility and trustworthiness must be critically assessed. At this purpose, Trust and Reputation Systems have been widely used for various ecommerce applications in order to assess the credibility and trustworthiness of the provided reputation information. Furthermore, this task is becoming increasingly important for the majority of eservices, but especially for e-commerce platforms where resources and business benefice value depend on making correct decisions. As a matter of fact, deliberately providing fake and dishonest ratings and reviews is a serious and crucial security issue that threaten the trust establishment and propagation in e-commerce environment. In fact, this misbehaving attitude would systematically falsify the trust and reputation assessment of the reviewed products and services in e-commerce applications. As a result, this falsification would impact customers' trust with regards to the purchase decisionmaking. Moreover, human users have specific reasons for deliberately skewing their comments and they can change their intentional behavior over time and according to changing circumstances that impact the product's quality 
and the customer's interests as well. Besides, customers can also be discriminative against particular service providers while cooperating with others.

In order to distinguish honest reputation information from dishonest one, we need a robust TRS that applies intelligent detection algorithms, either supervised, unsupervised or semi-supervised. These algorithms aim to analyze the trustworthiness of the reputation information provided in the form of reviews, recommendations and numeric ratings. In fact, a meticulous trustworthiness analysis of the shared information achieved by TRS, would certainly increase the system's robustness and resistance against fraudulent reviewers. Moreover, the underlying computational goal of TRS is to generate a trustful evaluation of the reputation of a product or a service in ecommerce. Indeed, TRS incarnate a combination of two dependant systems: Reputation systems which is generally based on the rating process, where entities rate each other after the achievement of a transaction [1]. Therefore, an algorithm is implemented in order to use the aggregated ratings about a specific entity to generate somehow a reputation score. On the other hand, Trust systems generate trust measures and scores according to the analysis of combined paths and networks of trust relationships between transacting peers $[1,2]$. Reputation scores and trust measures can be combined and derived from a TRS and thereby assist ecommerce users in their transaction and purchase decision making process.

Additionally, TRS analysis must focus on the review provided in the form of text sentence and the numeric score, rating or star rating proposed by the e-commerce strategy of rating, since it disseminate important latent and detailed reputation opinion on specific features of the reviewed product. Relying on Natural Language Processing (NLP) techniques inspired by intuitive human intelligent reflexes and processing, we believe that it is possible to build robust TRS able to create a trusted reputation management network that supports ecommerce applications, boosts their ability performances and reaches the intended business value.

Accordingly, we need to analyze the maturity of current TRS in order to extract their weaknesses and strengths. At this purpose, we consider that a TRS is mature, if a number of credibility aspects and criteria are verified by the System's approach. To gather these trustworthiness aspects, we have focused on more recent surveys on the TRS as well as novel TRS works such as [1, 3, 4] published in 2007, [5, 6, 7] in 2010, [8, 9, 10] in 2013, $[11,12,13]$ in 2013 , and $[14,15,16]$ in 2015 . The most relevant trustworthiness aspects that are the most used in the state of the art of the TRS are related to the reviewing and the rating process adopted in the TRS analysis.
Additionally, the selection of recommenders is also considered an interesting trustworthiness criterion that can explicitly impact the trustworthiness and reputation evaluation. In addition, the interpretation and the reasoning on the gathered information remains an indispensible trustworthiness criterion that explicitly acts on the computational approach to calculate the reputation value of the reviewer and the topic reviewed as well. At this purpose, we have adapted these three trustworthiness aspects to our purpose in order to establish a comparative study of a selection of the most popular and studied TRS. Besides, we have extended the comparison framework by adding other trustworthiness aspects. The first one is related to the computation approach to calculate and evaluate the trust and reputation. In fact, there are different computational approaches that can be either based on probabilistic methods to calculate the trust value, or on the Bayesian approach, or on fuzzy rules, statistical methods that derives a percentage representing the trust and reputation estimation, or Dirichlet reputation systems [3], etc... This aspect aims to present and describe reputation computation engines and approaches employed and/or enhanced to compute the reputation and trust value. Basing on this credibility aspect, we can analyze the trust and reputation evaluation results and then critically evaluate the reached accuracy-level of each TRS. In fact, the second trustworthiness aspect is the evaluation of the effectiveness of each TRS involved in the comparative study. Additionally, since text reviews are important in the TRS analysis, we compare the selected TRS in terms of their reliability on opinion or sentiment mining techniques especially for extracting the intended sentiment orientation from text reviews.

In this paper, we present in depth comparative study of the most popular, used and analyzed TRS according to the trust and reputation literature and in terms of specific trustworthiness criteria. We start introducing the chosen TRS for the comparative study. We then compare the TRS in term of the selected trustworthiness criteria. This survey aims to analyze and evaluate their maturity and effectiveness, especially regarding the computational approach employed to generate reputation and trust values. Additionally, we provide a summary table of the compared TRS within a detailed selection of trustworthiness criteria.

\section{Overview of the main TRS}

When considering online trust and reputation systems, eBay is both well researched and much written about [17, $18,19,20,21]$. eBay is an online auction site, allowing sellers and buyers to trade goods through an auction 
process. The commercial eBay TRS stores users' ratings linked to their profiles and related transactions, but leaves the credibility analysis to the human user. Unitec is also clearly directed towards a human user, but it performs automated credibility analysis as well. The content of a recommendation is not fixed, and the system could handle product recommendations with the same algorithm as well [1]. A number of TRS are designed for e-commerce applications such as FuzzyTrust [24, 49] and REGRET $[25,51]$ which are both designed for multi-agent marketplaces, but they apply different approaches to reputation estimation than ebay's TRS [1]. In fact, in FuzzyTrust, local trust scores are generated through fuzzy inference and aggregated to global reputation values. However, REGRET is a decentralized trust and reputation model designed for more complex e-commerce environments where various types of agents with different social relationships play important roles. With the help of a social structure called Sociogram, it is able to model the social relationships such as cooperation, competition and trade in a graph where the nodes represent the participants and the edges denote the nature of their relationship.

In other words, REGRET reputation system is designed to operate within an electronic marketplace settings relying on multiple contextual attributes to classify information as coming from an individual, social, or an ontological dimension. The individual dimension considers information directly gathered from interactions between two entities. The information is fine grained and often related to the frequency of overcharging, late delivery and quality of the transaction [7, 25].

For cooperative applications on the Internet, NICE is a good TRS example. Trustors are given signed receipts of successful transactions, "cookies", as a sign of some trust. These can be used to link actors into a weighted trust chain.

For peer-to-peer ecommerce communities, PeerTrust [28, $15,39]$ is a coherent dynamic trust model with unique characteristics tailored. This TRS uses a structured peerto-peer (P2P) overlay network to host a distributed implementation of their transaction-based feedback system [46]. The simulation used to demonstrate PeerTrust utilizes P-Grid to distribute feedback scores. The system incorporates a combination of fundamental reputation sources, such as direct feedback, and the quantity of transactions performed, while weighting feedback with credibility [1,7].

For this purpose, PeerTrust defines the personalized similarity measures, which compute feedback similarity rate between the evaluating peer and opinion providers over a common set of peers with whom they have had previous interaction. In fact, this TRS attempts to create trustworthy peers that consistently act honestly as a role of feedback provider, and do not become affected by malicious intentions such as jealousy and negative competitive attitude. Besides, this model assumes that the trust metric can be alternatively served as a credibility measure under certain circumstances $[1,7,43]$.

MDNT, Managing Trust and MLE [27, 42, 21, 22] topeer community environments, which cover both multiagent marketplaces and cooperative applications. In fact, they can host a multitude of activities especially in distributed applications such as information exchange (file transfers) and transactions in e-commerce applications [1, 7]. Furthermore, their approaches to compute reputation are varying as well.

As previously discussed, file transfers and sharing is a frequent activity in internet requiring security and trustworthiness verification. Indeed, EigenTrust [44] is a reputation system for peer-to-peer file sharing. It relies specifically on a global, shared reviews and recommendations concerning reputation. Credibility analysis is used in calculating each global reputation value [1].

However, for a wide application of open systems such as Grid service that are not restricted to one activity case, TRAVOS [31, 52] (Trust and Reputation model for Agent-based Virtual Organizations) is developed to ensure high quality interaction between the participants. It exploits two information sources to assess the trustworthiness of the participants: Direct Interaction and Witness Observation.

To derive trust, this model relies greatly on its direct experiences and refuses to combine others' opinions unless they are really required [43]. In fact, TRAVOS aims to ensure good interactions between self-interested software agents in large scale open systems, such as the Grid. The agents provide interchangeable services, and reputation information is used to choose the most trustworthy partner. Its reputation expression is based on Beta probability distributions [1].

For Multi-agent systems MAS, Fire [33, 51] is designed to handle the bootstrapping problem of newcomers and filter out inaccurate reputation information. In addition, this TRS attempts to differentiate between dishonest and mistaken agents and provide compound reliability measures by employing a multi-criterion rating system.

Moreover, the proposed TRS by $\mathrm{Yu} \&$ Singh [35] is also suitable for MAS. The system proposes novel trust and referral network able to detect three models of deceptions. It provides credibility measures pertaining to each model 
and it is adapted to differentiate between agents having bad reputation or no reputation using DempsterShafter theory of evidence. As a result, both Fire and the TRS by yu \& singh support dynamism in open MAS [7, 25, 27].

A key benefit of the social dimension is that it allows new and unproven entities to bootstrap their trustworthiness by belonging to reputable groups. Alternatively, because the entire group's reputation is associated with the behavior of its members, it is pertinent for a group's members to moderate the behavior of those associated with them.

Concerning open dynamic environments, BRS [34, 52] remains a very suitable TRS that supports binomial and multinomial rating models and also addresses bootstrapping problem by considering the quality of community in the marketplace. The TRS provides iterated filtering algorithm which can effectively reveal deceptive intentions if the majority of participants act honestly. BRS utilizes longevity factor to discount ratings as time progress, enable participants as buyers and sellers to adaptively change their behavior in order to increase their own benefits [7].

To summarize the overview of TRS selected for the comparative study, we propose the Table III.1 bellow that is an extension of the state of the art presented in [1] representing each TRS, its pertaining domain of application and the different actors involved in the Reviewing and Rating process. In fact, we have updated the related references and added the related information of the following TRS: Fire, BRS, TRAVOS and the TRS proposed by Yu and Singh.

Table 1:TRS and their Application Area

\begin{tabular}{|c|c|c|c|}
\hline TRS Name & References & Application Domain & Actors \\
\hline eBay & $\begin{array}{l}{[55] 2006} \\
{[46] 2010} \\
{[47] 2015}\end{array}$ & electronic Marketplace & human \\
\hline Unitec & $\begin{array}{l}{[38] 2003} \\
{[48] 2014}\end{array}$ & generic framework & human \\
\hline FuzzyTrust & $\begin{array}{l}{[24] 2005} \\
{[49] 2012}\end{array}$ & $\begin{array}{l}\text { multi-agent } \\
\text { marketplace }\end{array}$ & agents \\
\hline REGRET & $\begin{array}{l}{[25] 2002} \\
{[51] 2015}\end{array}$ & $\begin{array}{l}\text { multi-agent } \\
\text { marketplace }\end{array}$ & agents \\
\hline NICE & $\begin{array}{l}{[26] 2003} \\
{[50] 2012} \\
{[5012}\end{array}$ & $\begin{array}{l}\text { cooperative } \\
\text { applications }\end{array}$ & agents \\
\hline MDNT & $\begin{array}{c}{[27] 2004} \\
{[43] 2014}\end{array}$ & online communities & agents \\
\hline PeerTrust & $\begin{array}{l}{[28] 2004} \\
{[15] 2015}\end{array}$ & online communities & agents \\
\hline Managing Trust & $\begin{array}{l}{[28] 2001} \\
{[51] 2015}\end{array}$ & online communities & Agents \\
\hline MLE & $\begin{array}{c}{[28] 2004} \\
{[53] 2014}\end{array}$ & online communities & agents \\
\hline EigenTrust & $\begin{array}{c}{[31] 2003} \\
{[54] 2015}\end{array}$ & file sharing & agents \\
\hline
\end{tabular}

\begin{tabular}{|l|c|l|l|}
\hline TRAVOS & $\begin{array}{c}{[32] 2006} \\
{[52] 2014}\end{array}$ & Grid services & Agents \\
\hline FIRE & $\begin{array}{c}{[33] 2006} \\
{[51] 2015}\end{array}$ & Multi-agent Systems & Agents \\
\hline BRS & $\begin{array}{c}{[34] 2002} \\
{[52] 2014}\end{array}$ & $\begin{array}{l}\text { Multi-agent } \\
\text { marketplace }\end{array}$ & human \\
\hline TRS by yu \& singh & $\begin{array}{c}{[35] 2003} \\
{[43] 2014}\end{array}$ & Multi-agent Systems & Agents \\
\hline
\end{tabular}

\section{Comparing trustworthiness criteria of the} main TRS

In the previous section, we have presented a brief overview of the most used and researched trust and reputation systems in their different areas of application such as in e-commerce communities, multi-agent systems, Grid services, cooperative applications, peer-to-peer marketplaces...etc. Each one of the presented TRS uses different Reputation evaluation approach according to the effectiveness perspectives.

In fact, a number of trustworthiness aspects must be verified in order to analyze the robustness and effectiveness of the TRS. In the coming sections, we will first discuss the taxonomy of the selected credibility aspects and then compare TRS within a critical study using aspects of trustworthiness.

In order to summarize the comparative study realized on the main used TRS, we selected the most distinguishing trustworthiness criteria previously described.

We also propose to extend some of the trustworthiness criteria in order to derive interesting conclusions from this summarization. For this purpose, we propose to synthesize the trustworthiness criteria involved in the analysis in six different categories as follows:

-1) Reviewing strategy: This category involves criteria that recapitulate the rating and reviewing process adopted by a specific TRS. The "text review" indicates the possibility of writing a text opinion expressing sentiments, emotions and disseminating recommendations about a target topic. Another interesting criteria is the category of available and stored reviews: whether the system considers both "positive and negative" reviews or only one of the two.

-2) Analysis \& interpretation of the provided reviews: In the case of a system authorizing both text reviewing and numeric rating, the reputation computation approach must first of all verify the "concordance" between the text review and the numeric score. 
Depending on specific circumstances, some of these TRS "combine" the two types of ratings in the reputation evaluation to derive trust and others solely focus on one category for less complex reputation calculations. Nevertheless, it is still necessary to automatically extract sentiments and opinions expressed in the text reviews. For this purpose, "opinion mining" techniques must be applied to mine the latent sentiments and opinions provided in text reviews.

-3) Influencing parameters in the reputation evaluation: According to the comparative study achieved in the previous section, we have considerably noticed that the reputation computation approach mainly relies on a number of influencing parameters that significantly impact the reputation and trust assessment. These parameters represent mainly "transaction context factors", "history record" and "time" information. Accordingly, we compared the selected TRS based on these trustworthiness aspects as shown in Table 2.

-4) Reputation interpretation \& update: The "interpretation of a reputation value" and its eventual update represent crucial comparison aspects that systematically impact the effectiveness of a TRS. Trust and reputation are generally regarded as feelings that purely rely on human cognitive faculties. Nonetheless, these concepts are to be computationally formalized in order to be involved in the trust and reputation computation -engine. For this purpose, the computational interpretation of the trust and reputation values and the appropriate selection of thresholds are very important and can considerably influence on the TRS effectiveness. Besides, the "reputation update process" is more likely neglected by a great number of TRS. This process involves in and of itself a selection of criteria that control the time of update, the update reasons and its frequency.

-5) Malicious \& deceptive agents' control: Agents and reviewers are the available information resources in any TRS. Their shared information is commonly relied on by other recommendations' requesters. However, "malicious reviewers" deliberately disseminate misinformation through the network so as to falsify the reputation value. Accordingly, the effectiveness of the TRS relies on its adopted approach to detect, "control" and deal with these deceptive recommenders. For this purpose, the effectiveness of a TRS to encounter malicious agents and control their participations was employed as a relevant trustworthiness criterion in the comparative study.

-6) Witness observation \& the reputation evaluation: Some of the compared TRS apply the witness observation and opinion as a complementary information module to enrich the information so as to derive an overall reputation estimation of the observed entity. Depending on the TRS testimony policy, the identity of reviewers can either be revealed, or kept private and "confidential". Besides, the TRS can opt for the complete anonymity of recommenders as Managing Trust does.

TABLE 2 Summary of the TRS analysis within a set of credibility criteria

\begin{tabular}{|c|c|c|c|c|c|c|c|c|c|c|c|c|}
\hline IRS Name & $\begin{array}{l}\text { Text } \\
\text { review }\end{array}$ & \begin{tabular}{|l|} 
positive \\
and \\
negative \\
review \\
\end{tabular} & $\begin{array}{l}\text { Combinat- } \\
\text { ion of } \\
\text { rating and } \\
\text { text } \\
\text { review }\end{array}$ & \begin{tabular}{|l|} 
Concordance \\
betwiven \\
rating and \\
text revielli \\
\end{tabular} & \begin{tabular}{|l|} 
Transact \\
- ion \\
context \\
factors \\
\end{tabular} & $\begin{array}{l}\text { History } \\
\text { Record }\end{array}$ & \begin{tabular}{|l|} 
time \\
factor \\
\end{tabular} & \begin{tabular}{|l|} 
Reputation \\
Value \\
\end{tabular} & $\begin{array}{l}\text { Reputation } \\
\text { value } \\
\text { update }\end{array}$ & $\begin{array}{l}\text { malicious } \\
\text { agents } \\
\text { Control }\end{array}$ & \begin{tabular}{|l|} 
Third-part \\
witnesses \\
observation
\end{tabular} & $\begin{array}{l}\text { Reviemer's } \\
\text { confidence }\end{array}$ \\
\hline eBay & yes & $\begin{array}{l}\text { only } \\
\text { positive }\end{array}$ & No & No & No & yes & No & $\begin{array}{l}\text { statisitics, } \\
\text { text }\end{array}$ & No & No & No & yes \\
\hline Unitec & yes & yes & $\begin{array}{l}\text { Not } \\
\text { mentioned }\end{array}$ & No & No & No & $\begin{array}{l}\text { possibl] } \\
y\end{array}$ & $\begin{array}{l}\text { configuabl } \\
e\end{array}$ & Yes & No & No & yes \\
\hline FuzzyIrust & No & yes & No & $\begin{array}{l}\text { No terst } \\
\text { review }\end{array}$ & yes & yes & yes & numeric & Yes & yes & No & No \\
\hline REGRET & yes & yes & yes & No & yes & yes & yes & $\begin{array}{l}\text { numeric, } \\
\text { categonized }\end{array}$ & Yes & yes & yes & yes \\
\hline NCE & yes & yes & $\begin{array}{l}\text { Not } \\
\text { mentioned }\end{array}$ & No & yes & yes & yes & $e^{\text {configurabl }}$ & Yes & No & yes & No \\
\hline MDNT & yes & yes & No & No & yes & yes & yes & $\begin{array}{l}\text { numeric, } \\
\{0, \ldots, 6\}\end{array}$ & Yes & No & No & No \\
\hline Peer'Tust & yes & yes & yes & No & yes & yes & yes & $\begin{array}{l}\text { numeric, } \\
{[0,1]}\end{array}$ & Yes & yes & yes & No \\
\hline $\begin{array}{l}\text { Managing } \\
\text { Trust }\end{array}$ & yes & \begin{tabular}{l|} 
only \\
negative
\end{tabular} & No & No & No & yes & No & $\begin{array}{l}\text { numeric, } \\
1,0,1\end{array}$ & Yes & No & jes & jes \\
\hline MLE & No & yes & No & $\begin{array}{l}\text { No text } \\
\text { review }\end{array}$ & No & yes & No & probability & Yes & No & yes & yes \\
\hline EigenInust & yes & yes & No & No & No & yes & No & $\begin{array}{l}\text { stratistics, } \\
\text { text }\end{array}$ & Yes & yes & No & No \\
\hline Travos & yes & yes & yes & No & No & yes & No & probability & Yes & No & yes & No \\
\hline FIRE & jes & yes & yes & No & yes & jes & $\begin{array}{l}\text { possibl } \\
y\end{array}$ & $\begin{array}{l}\text { multi- } \\
\text { criterion } \\
\text { rating }\end{array}$ & Yes & jes & yes & No \\
\hline BRS & No & yes & No & $\begin{array}{l}\text { No text } \\
\text { review }\end{array}$ & yes & yes & yes & $\begin{array}{l}\text { biromial } \\
\text { and } \\
\text { multinomia } \\
\text { lratings }\end{array}$ & Yes & jes & No & yes \\
\hline $\begin{array}{l}\text { TRS by Yu } \\
\text { and Singh }\end{array}$ & yes & yes & yes & No & yes & yes & yes & $\begin{array}{l}\text { Dempsters } \\
\text { hafter } \\
\text { theory of } \\
\text { eridence }\end{array}$ & Yes & jes & yes & jes \\
\hline
\end{tabular}

The selected TRS are compared based on these trustworthiness criteria and summarized in the following table. As shown in Table 2, none of the main TRS uses opinion mining approaches to semantically analyze text opinions. Moreover, a considerable number of these TRS do not involve text reviews in the reputation computation 
engine and instead they only rely on the overall numeric rating. Besides, time factor and history record, which noticeably impact the TRS effectiveness, are not considered in several TRS. Furthermore, for TRS involving text reviews in the reputation evaluation process, they neglect the concordance verification between the overall numeric rating and the text review. This concordance reveals the similarity or dissimilarity between the two related values.

\section{Conclusion}

Since last decade, the application and usage of TRS in eservices especially in the e-commerce environment, have fascinated many research attentions around the globe. Various research efforts attempt to design and implement a robust TRS able to detect and encounter malicious interactions, deceptive users and to assess and propagate trust among online communities. Furthermore, a number of TRS intend to generate a most reliable reputation estimation related to the review and the reviewer as well. According to the achieved synthesis, we notice that the majority of the compared TRS encourage their users to write text reviews. Indeed, most of online users rely more on text reviews rather than numeric ratings, because opinion expressions are more expressive and summarizing for a recommendation requester. Furthermore, text opinions recapitulate interesting and opinion-rich real-life experiences, so that the user could gain different perspectives towards the target product.

Opinion mining or sentiment mining is the most relevant NLP technique that attempts to mine text recommendations, in order to detect and extract the recommender's intention toward the reviewed product or the service. In addition, opinion mining aims to accurately predict and compute the sentiment orientation polarities of the reviewed products' features and subfeatures as well. Most recent papers and future work would be devoted to the inclusion of NLP techniques in order to boost TRS performance by exploiting text reviews expressed by reviewers. This text reviews analysis might reveal reviewers sentiments and intentions towards reviewed products and hence increase TRS robustness, effectiveness and intelligence.

\section{References}

[1] A. Jøsang. Trust and Reputation Systems. Appears in A. Aldini and R. Gorrieri (Eds.), Foundations of Security Analysis and Design IV, FOSAD 2007 Tutorial Lectures. Springer LNCS 4625. ISBN 208-3-540-7109-0. Bertinoro, Italy, September 2007.

[2] H.Rahimi and H. El Bakkali. "A New Trust Reputation System for E-Commerce Applications". In the proceedings of the International Journal of Computer Science Issues IJCSI, 2014.

[3] A. Jøsang, R. Ismail, and C. Boyd, A survey of trust and reputation systems for online service provision, Decision Support Systems, vol. 43, no. 2, pp. 618-643, 2007.

[5] Huanyu Zhao and Xin Yang Xiaolin Li: WIM: A Wagebased Incentive Mechanism for Reinforcing Truthful Feedbacks in Reputation Systems. In the Proceedings of the Global Communications Conference. GLOBECOM, 6-10 December 2010, Miami, Florida, USA. IEEE.

[6]Félix Gómez Mármol, Joao Girao, Gregorio Martínez Pérez: TRIMS, a privacy-aware trust and reputation model for identity management systems. In the proceeding of Computer Networks, 54(16):519-2912, September 2010.

[7] Zeinab Noorian and Mihaela Ulieru. The State of the Art in Trust and Reputation Systems: A Framework for Comparison. In the proceedings of the Journal of Theoretical and Applied Electronic Commerce Research ISSN 07181304, VOL 5, ISSUE 2, AUGUST 2010, pp: 20-117.

[8] Zhang, Y., Zhu, W. Extracting implicit features in online customer reviews for opinion mining. In the Proceedings of The 22nd International Conference on World Wide Web companion. International World Wide Web Conferences Steering Committee, 2013, pp. 42-43.

[9] Jens Lehman, Robert Isele, Max Jakob, Anja Jentzsch, Dimitris Kontokostas, Pablo N. Mendes, Sebastian Hellmann, Mohamed Morsey, Patrick van Kleef, Sören Auer, Christian Bizer. DBpedia - A Large-scale, Multilingual Knowledge Base Extracted from Wikipedia. In the proceedings of the WWW2013 Workshop on Linked Data on the Web, Rio di janeiro.

[10] B.Min. Distant Supervision for Relation Extraction with an Incomplete Knowledge Base. In the Proceedings of NAACLHLT, 2013 [11] Audun Jøsang, Touhid Bhuiyan, Yue $\mathrm{Xu}$, and Clive Cox. Combining Trust and Reputation Management for Web-Based Service. In the proceedings of the 5th International Conference on Trust, Privacy \& Security in Digital Business (TrustBus2008), Turin, September 2008.

[12] Khairullah Khan, Baharum Baharudin, and Aurangzeb Khan. Identifying Product Features from Customer Reviews Using Hybrid Patterns. In the proceedings of The International Arab Journal of Information Technology, Vol. 11, No. 3, May 2014.

[13] Y. Zhang, Min Zhang, Yiqun Liu and Shaoping Ma. Do users rate or review?: boost phrase-level sentiment labeling with reviewlevel sentiment classification. In the Proceedings of the 37th international ACM SIGIR conference on Research \& development in information retrieval. Pages: 417-420, ACM 2014, New York, NY, USA. [14] Maryam Saeedi, Zeqian Shen and Neel Sundaresan The Value of Feedback: An Analysis of Reputation System. In the proceedings of the international February 17, 2015.

[15] Vinaya R. Firake, Yogesh S. Patil. Survey on CommTrust: Multi-Dimensional Trust Using Mining ECommerce Feedback Comments. In the proceedings of the International Journal of Innovative Research in Computer and Communication Engineering IJIRCCE 
10.15628/ijircce.2015.0303037 1640, Vol. 3, Issue 3, March 2015 .

[16] Njagi D. Gitari, Z. Zuping, H. Damien, and J. Long. A Lexicon-based Approach for Hate Speech Detection. In the proceedings of the International Journal of Multimedia and Ubiquitous Engineering, April 2015, Vol.10, pp.52230.http://dx.doi.org/10.14257/ijmue21. ISSN: 12050028.

[17] M. Kinateder, K. Rothermel, Architecture and algorithms for a distributed reputation system. In the Proceedings of The First International Conference on Trust Management, Springer-Verlag, 2003, pp. 1-16. FOR TEXTUAL FEEDBACK [46] P. Resnick, K. Kuwabara, R. Zeckhauser, E. Friedman. Reputation systems. In the Proceedings of The Commun. ACM 43 (2000) 45-1.

[18] P. Resnick, R. Zeckhauser, Trust among strangers in internet transactions: Empirical analysis of eBay's reputation system. In the Proceedings of The Advanced Microeconomics: An Annual Research 11 (2002) 15-157.

[19] P. Resnick, R. Zeckhauser, J. Swanson, K. Lockwood, The value of reputation on eBay: A controlled experiment. In the Proceedings of The Experimental Economics 9 (2003) 27-40.

[20] D. Houser, J. Wooders, Reputation in auctions: Theory, and evidence from eBay. In the Proceedings of The Journal of Economical Management Strategy 15 (2) (2006) 353369.

[21] M.I. Melnik, J. Alm, Does a Seller's eCommerce reputation matter? Evidence from eBay Auctions. In the Proceedings of The Journal of Industrial Economics 50 (3) (2002) 337-349.

[22] P. Resnick, R. Zeckhauser, J. Swanson, and K. Lockwood, The value of reputation on eBay: a controlled Experiment. In the Proceedings of The Esa Conference, Boston, Ma, 2002.

[24] S. Song, K. Hwang, R. Zhou, and Y.-K. Kwok. Trusted P2P transactions with fuzzy reputation aggregation. In the Proceedings of the IEEE Internet Computing, 9(6):24-33, 2005.

[25] J. Sabater and C. Sierra. Reputation and social network analysis in multi-agent systems. In the Proceedings of the AAMAS '02: the First International Joint Conference on Autonomous Agents and MultiAgent Systems, pages 438$12,2002$.

[26] S Lee, R. Sherwood, and B. Bhattacharjee. Cooperative peer groups in NICE. In the proceedings of the International Journal of Computer and Telecommunications NetworkingManagement in peer-to-peer systems, ACM digital Library, 2006, pages: 523-544. [27] S. Staab et al. The pudding of trust. In the Proceedingsof the IEEE Intelligent Systems, 19(5):55-36, 2004.

[28] L. Xiong and L. Liu. PeerTrust: Supporting reputation based trust for peer-to-peer electronic communities. In the Proceedings of the IEEE Transactions on Knowledge and Data Engineering, 16(7):323-337, 2004.

[29] K. Aberer and Z. Despotovic. Managing trust in a peer-2-peer information system. In Proceedings of the 10th International Conference on Information and Knowledge Management (2001 ACM CIKM), Atlanta, 2001.
[30] Z. Despotovic and K. Aberer. Maximum likelihood estimation of peers' performance in P2P networks. In the Proceedings of the The Second Workshop on the Economics of Peer-to-Peer Systems, 2004.

[31] S. Kamvar, M. Schlosser, and H. Garcia-Molina. The EigenTrust algorithm for reputation management in P2P networks. In Proceedings of the Twelfth International World-Wide Web Conference (WWW03), pages 446-458, 2003. 